\title{
Media archaeology-based Visual Music ${ }^{1}$
}

\author{
Alberto Novello \\ Submitted 14th February 2021 \\ Revision 1st June 2021
}

\section{Introduction}

An official definition of Visual Music as an art form is still somehow debated and unclear (Garro 2012). However in the last decades several worldwide exhibitions have been inspired by the idea of connecting sound and light under the common name of Visual Music. To date, scholars have proposed different perspectives of analysis: McDonnel considers a set of compositional practices adopted from music and translated into visual arts (McDonnel 2007); DeWitt approaches the psychological and aperceptual effects on the observer-listener (deWitt 1987); Evans codifies the rules of "visual dissonance and consonance" as extensions of the concepts of tension and resolution from tonal harmony in music (Evans 2005). Friedlander condenses the complex set of works of visual music into three possible categories: the translation of music into image following a set of rules executed by machines or algorithms, the conversion operated by an artist through free interpretation, and music-inspired imagery, where the presence of a sonic event is not necessary but just inspirational (Friedlander 2021). After curating one of the largest exhibitions of Visual Music in 2005, Jack Ox and Cindy Keefer, both experts and active lecturers in the field of Visual Music, synthesized the amount of work submitted to the open call into a phenomenological definition of Visual Music in four categories (Ox et al. 2008): the translation of a piece of music into images, usually defined as Intermedia ${ }^{2}$; a time-based narrative with a visual structure, where light movements are intended as music events and distributed over time, with or without sound; a visual composition created in a linear way following a time development but rather static, as on a canvas; the direct, non-interpretative translation of images to sound or vice-versa: 'what you hear is exactly what you see'3.

1 Supplementary materials to this article (images and Max/MSP Patches), all marked with bold words in the text, can be found online at the following DOI: 10.5281/zenodo.4761423.

${ }^{2}$ Dick Higgins first defined Intermedia as the combination of structural elements from two or more different media into one medium (Higgins 2001).

3 Ox, J. and Keefer C., On Curating Recent Digital Abstract Visual Music, http://www.centerforvisualmusic.org/Ox_Keefer_VM.htm (all websites were visited on March 2021). 
Most diffused Visual Music definitions often invoke the concept of synesthesia in an attempt to vaguely depict the interexchange and connection between visual and auditory experiences. Psychologists identify synesthesia as a specific condition that occurs when an individual, receiving a stimulus in one sense, simultaneously experiences a sensation in another. In history, several composers have been influenced by synesthetic perceptions: Scriabin associated colors with the various harmonic tones of his atonal scale (Cytowic et al. 2009); Messiaen in his Traité des rhythmes, de couleurs et d'ornitologie, describes the connection of chords to specific colors (Bernard 1986); Lygeti was convinced that 'major chords are red or pink, minor chords are somewhere between green and brown' (Rogowska 2011). However in the art world, the word synesthesia has been widely misused and drifted from its original definition to identify any multimodal sensory experience such as live cinema or a video jockey show (Evers 2020, Holzer 2019).

In this article, I describe my personal artistic practice developed during the last seven years based on lasers, modified cathode ray tube (CRT) monitors, and oscilloscopes to create works following a strict and somehow objective definition of synesthesia in Visual Music: when the same untranslated signal is sent to deflect a light beam to create images and to drive the coils of loudspeakers which convert these signals into sound. I embrace the expressive limitations imposed by the direct translation of the same signal into light and sound in favor of the intrinsic gained coherence, avoiding any arbitrary juxtaposition of image and sound by the artist. Enveloping the audience in synchronous sound and light information reveals visually the underlying sound properties and geometries of sound, exposing to the eyes what is sometimes obscure for the ears: frequency ratios, phase shifts, detuning and beatings, etc. I call this process visual listening: a deeper way of understanding sound through its visualization through light. Despite the tradition of defining Visual Music using other art forms, I see this practice as self standing, possessing its own methodology and aesthetic. Because of the perfect audiovisual synchronization, the audience instantaneously understands the rules at play and enjoys the limitations and risks taken by the artist.

My practice is based on resurrecting and repurposing old media for the creation of live performances and installations. This knowledge is distributed to the community in the form of lectures, workshops and free software. I find the vibrance of light, the infinite resolution, and the absence of frame rate of the light beam of a CRT monitor or laser more appealing than the ubiquitous digital projections. Besides the analog aesthetics, I also consider the environmental impact and the charm of repurposing obsolete devices as an archaeological media. Ultimately this choice helps me to differentiate my practice from the mainstream of digital computer art. By exposing the public to the aesthetic differences between old and new devices, I invite them to reflect on the sociopolitical impact of technology, in a retrospective on technologization: what old means, and what value the new really adds. I do not discard but embrace hybridization by combining the advantages of both eras: the fluidity and vibrancy of colors of analog light beams and the precision and replicability of numeric control. I believe that a device that has been revived and hybridized in this way is capable of generating new aesthetic experiences for the audience. 


\section{The aesthetic of visual music}

The connection between sound and visual arts was already studied by Pitagora in his theory of proportions, successively in Plato's Timaeus, and Aristotle with the first theory of correspondence between sound and color (Abbado 2018). However, only with the observations by Sir Isaac Newton on dividing sunlight into primary colors and color mixing in the book Opticks (1704), a systematic experimentation and theorization of light started to diffuse throughout Europe. A concrete application of such experiments is Castel's Clavecin Oculaire (Ocular Harpsichord) of 1725, the first instrument to simultaneously generate colors and sounds (van Helden et al. 1994). The tradition of color organs continued till the 19th and 20th century: from mechanical to electric organs, then to the cybernetic Musicolor Machine conceived by Gordon Pask, and eventually to the computer-based machines for the simultaneous creation of light and sound (Pickering 2011).

Parallel to these technological inventions, painters such as Kandinsky, Mondriaan and Klee found in sound an inspiration to free colors from the constraints of representation. The creation of abstract shapes followed an imaginary narrative on the canvas, similar to the practice of music composition on a score (Abbado 2018). Several music composers followed the opposite direction: composing music for visual events such as Handel's Music for the Royal Fireworks or Scriabin's Prometheus: The Poem of Fire, accompanying the orchestra with a Clavier a Lumiers called Chromola (Dann 1998).

The invention of film, with the consequent possibility to compose time in moving images, drew the most attention at the beginning of the 20th century. The first works, now lost, belong to Italian Futurists Arnoldo Ginna and Bruno Corra in 1910 (Ox et al. 2008). However, it was in Germany in the '20s that Hans Richter, Viking Eggeling, Walther Ruttmann, and Oskar Fischinger, created most of the early experimental audiovisual movies. Fischinger, one of the most prolific authors of the first half of the century, developed abstract movies using original techniques such as the Wax-Slicing Machine and the Lumiagraph, employed for public audiovisual live performances.

In the United States, several technological inventions allowed John and James Whitney to reach new aesthetics in their abstract movies. The pendulum sound device was used to generate waveforms that were imprinted on the optical soundtrack of some early experimental films. The motion cam machine, which pioneered the concept of motion control, later became the inspiration for the creation of the slit-scan machine built by Douglas Trumbull for the famous Stargate Corridor sequence of Stanley Kubrick's 2001: A Space Odyssey (Youngblood 1970). The use of early analog computers allowed the Whitneys to deterministically draw the image while capturing it, a tradition that evolved into early digital computer art, video synthesis tools, and contemporary artists writing their own software for digital computer animations such as Vsynth by Kevin Kripper and Hydra by Olivia Jack (Kripper 2021, Jack 2021).

In all these early works, the connection between sound and image is often arbitrary and compositional rules vary from piece to piece of each author's production depending on needs or means. The complete objectivation of the connection between sound and image through the same signal became possible with the technological shift from 


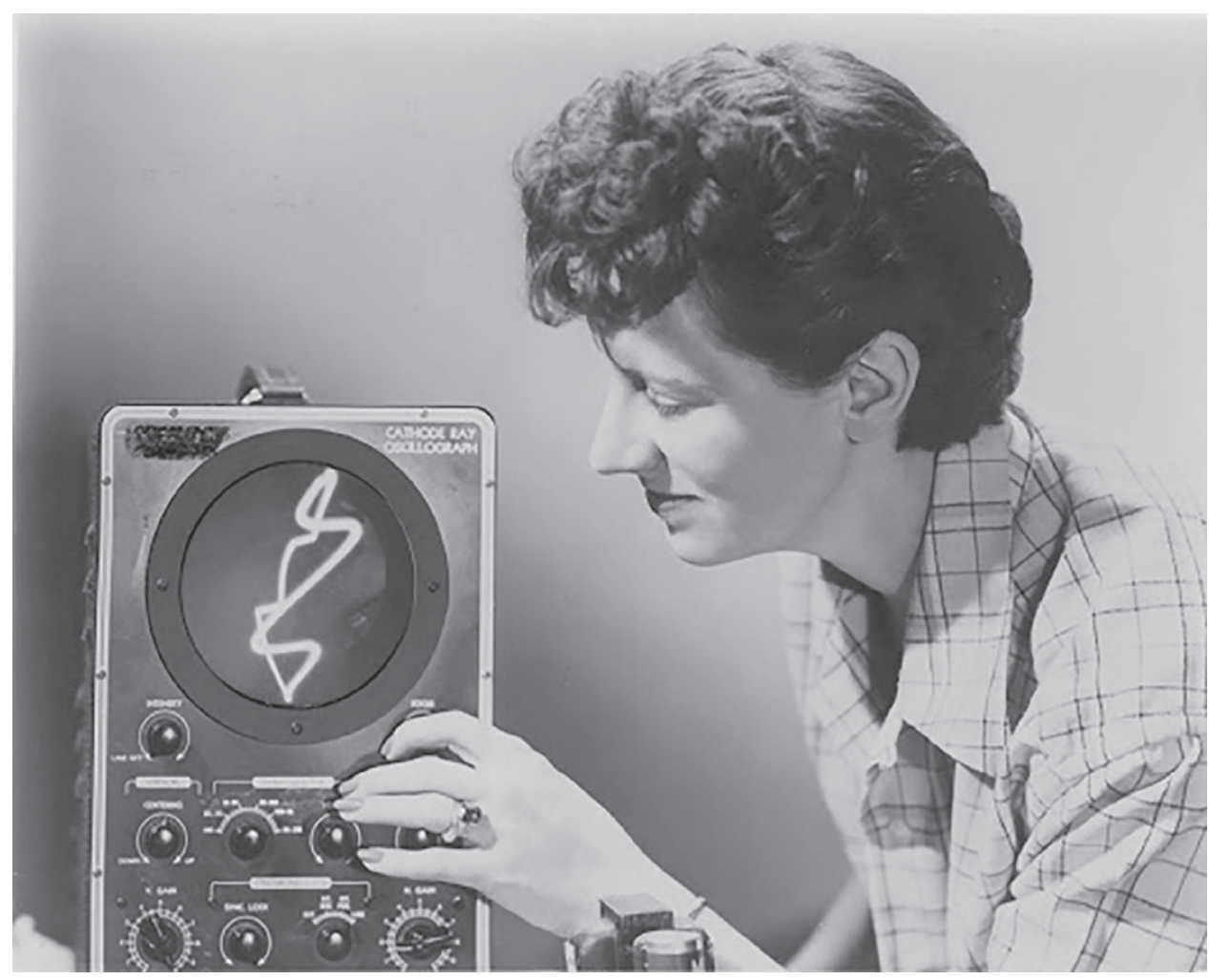

Figure 1a. Marie Ellen Bute - an early pioneer of experimental and animated film, using the same signal to draw images on oscilloscopes and drive loudspeakers to produce sound for her series of films called Seeing Sound. Courtesy Center for Visual music.

the gears of mechanical machines to the voltage signals of electromagnetic coils common to both monitors and loudspeakers. Mary Ellen Bute was probably the first artist to experiment in this direction using oscilloscopes to produce audiovisual works for her series of films called Seeing Sound in the '30s (Smirnov 2013). Norman McLaren used oscilloscopes and other techniques to achieve audiovisual synchronicity for example by projecting the celluloid optical soundtrack as visual projection. In the ' 50 s Ben F. Laposky modified oscilloscopes to expand the set of possible images obtainable with such devices. He named these images Oscillons.

In the '60s, the widespread diffusion of electronics in the arts contributed to the birth of the first analog sound synthesizers by Buchla, Moog and EMS. Applying the same principle of control voltage, the first video synthesizers could easily dialogue with audio synthesizers. Steina and Woody Vasulka largely experimented with the exchange of signals between audio and video domains. Built in the early '70s, Stephen Beck's Direct Video Synthesizer was essentially a modified TV set using audio signals to modulate the colors (Vasulka et al. 1992).

In the same period, the invention of lasers introduced the possibility of wide range projections of colored light controlled by electric signals. Artist Elsa Garmire, physi- 


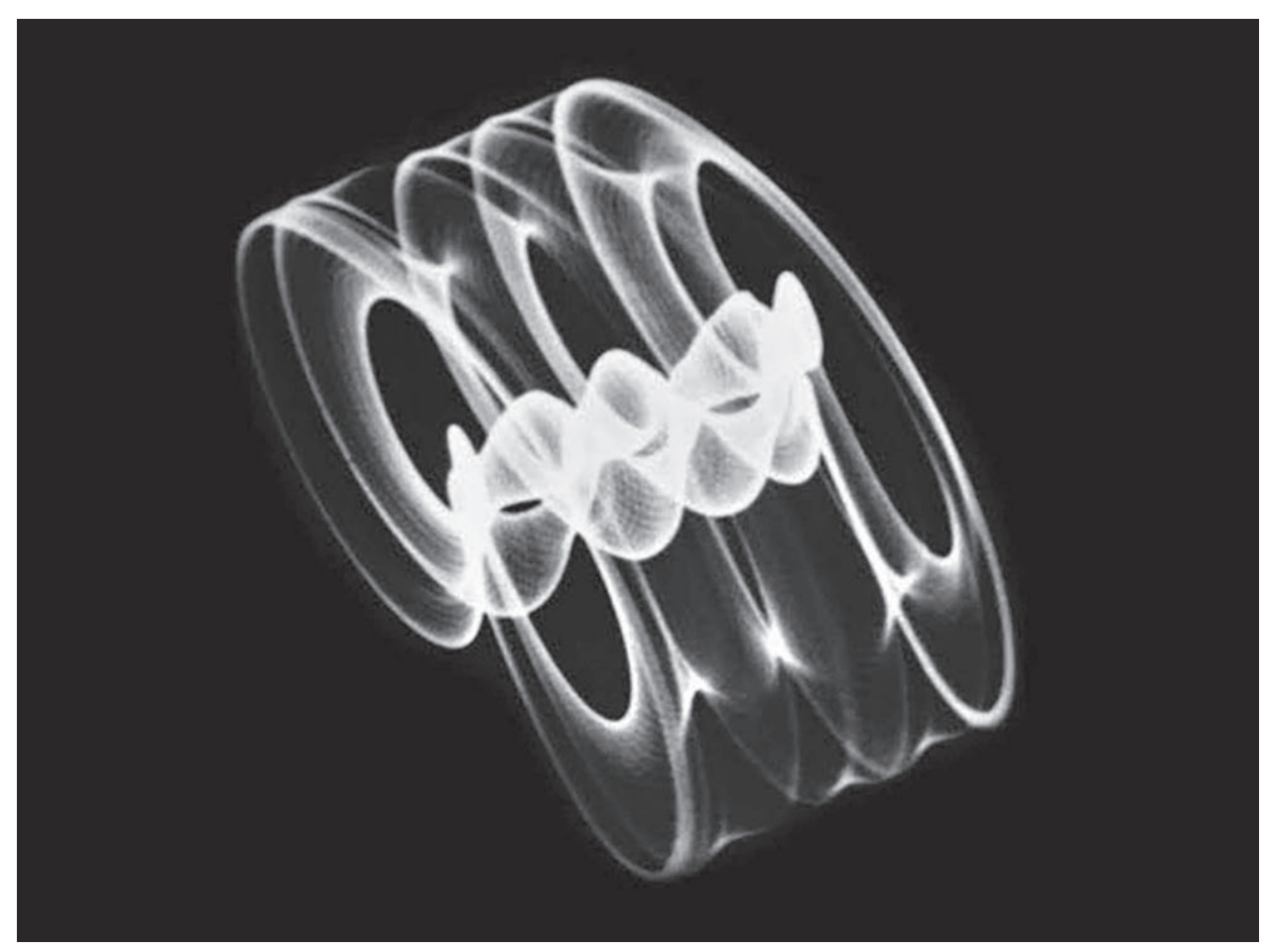

Figure 1b. Oscillon 21 - Ben F. Laposki (1953).

cist and co-founder of Laser Images, performed the first laser show in history to the music of Aaron Copland in 1973 (Shechet 2018). Ronald Pellegrino is the laser artist that early on saw the potential of a direct and untranslated sonification of the signals of the projected shapes through loudspeakers (Pellegrino 1983).

After a long pause during the ' 80 s and ' 90 s, modern synchronous audiovisual laser performances appeared again with the works of artists Robin Fox and Edwin van der Heide. Since 2007 Fox has used a laptop and a digital-to-analog converter to drive laser projections through haze. The laser-based performance is an extension of previous work undertaken using oscilloscopes to create audio-visual equivalence: BackScatter (2004) (Fox 2021). Edwin van der Heide in his work LSP (2008), focuses on the subject of composing signals that have both a structural musical quality and a time-based visual quality. Van der Heide uses lasers through haze to create three-dimensional changing environments that surround the audience (van der Heide 2021). More recently Robert Henke used lasers in several performances (Lumiere I, II and III), and installations such as Phosphor and Fragile Territories. Henke uses computers and signals from his soundcard to drive the lasers, however he always adds a different sound material to the laser shapes rather than using their original signal (Henke 2021).

The recent digitalization and the signal processing tools available in the arts allow new possibilities of decoupling the image from sound while keeping a synchronized workflow: each computing machine can be dedicated to the real-time rendering 
of one medium while exchanging event information with the others. Artists such as Ryoji Ikeda, Ryoichi Kurokawa or Carsten Nicolai applied these ideas to create reactive live shows (Ikeda 2021, Kurokawa 2021, Nicolai 2021). Fast last-generation computers are necessary to smoothly run real-time softwares for large installations and live animations.

Another result of the massive digitalization, the democratization and simplification of the software for audiovisual creation, determined a rapid growth of the VJ scene. Despite the creative possibilities offered by modern softwares, often the most important part of the VJ's work consists in the collection of video samples and the navigation among images, in which forms and rhythm seems more important than the images chosen themselves (Abbado 2018).

Possibly because of the over saturation of this field, several performers moved towards live cinema, in which image seems to become again an important part of the show, live coding, to expose the public to the code behind each sonic or visual element, or with a more material approach in what Zaarei calls Audiovisual Materialism (Zaarei 2020). Maybe as a reaction to the hasty race towards more modern technology, an increasing number of artists are redirecting their practices towards obsolete analog tools: video synthesizers, lasers, oscilloscopes, tape recorders, etc. After the celebration by the glitch aesthetic of the fallibility of the digital promises of precision and control, the intrinsic noise of discarded tools became suddenly compelling (Connolly et al. 2014). Analog tools inevitably carry a different aesthetic from the widespread digital machines: it is an experiential perception that requires no explanation. Besides the different qualities, the old tools remain fascinating because they embody the construction logic of the past generations. It is a journey backwards into the past, to rediscover what has been left behind and why, what type of mindset assembled those machines in that precise way, and to rethink technology and technologization.

\section{Media archaeology as a methodology}

Media archaeology interprets current human technology and emerging media through an analysis of the past, in particular by attempting to criticize the common narratives of a linear and deterministic progress of the evolution of human technology. One of the main fields of inspection is directed towards artistic production such as cinema and television (Parikka 2011). With particular attention to technological and scientific developments, we notice how evolution is not a linear nor a rational process and that «all excavations in the past shed light on our present» (Foucault 1972).

Every discontinuity in history creates new ideas, a paradigm shift and, as a consequence, the rejection of old ways of thinking and old tools that inevitably become dead media, as defined by the writer Bruce Sterling (Sterling et al. 2021). Media Archaeology widens the temporal scope of every object considering its environmental implications, realizing that old media never leaves; it either resurfaces as toxins in the soils or it is reinterpreted and reutilized, becoming zombie media: not alive but also not dead (Hertz et al. 2012). Zielinski suggests observing longer time scales than the 
«short term user values» proposed by corporations to assess the impact of our tools (Zielinski 2006).

In the last decades most of our media progressively lost their tangibility (digitized images, mp3 audio and streamed video calls), having disappeared into clouds of remote servers and internet services. Our control on information is slowly getting away from us, in miniature electronics inaccessible for our fingers and eyes. Cadoz warns us about the consequences of technological scales: each human advancement reduces the size of our tools, with the price of losing the instinctual understanding of the world at our hands (Cadoz 1988). The simplified user interfaces hide complicated backend softwares that only expert engineers are able to repair or control: substitution is better than reparation, the motto of planned obsolescence. Opposed to these ideas, a growing group of philosophers, artists, environmentalists, historians advocate for a retrospection on materiality. What can we do with old tools, in an overpopulated era in which recycling becomes essential, where most of the products we need are designed with planned obsolescence by corporations?

The history of music is dense with individuals developing or re-adapting past technology in new forms: Do-It-Yourself (DIY) culture, hardware hacking and circuit bending are based on repurposing old media and destining them to new lives and artistic purposes. The concept of hardware hacking permeated the beginnings of electronic music since the '60s with the works of John Cage (Bernstein 2015), Gordon Mumma, and the circuits of David Tudor (Tudor 2021). Nicolas Collins in his book Handmade Electronic Music provides practical examples of resurrecting all types of old media, repurposing discarded telephone coils, credit card readers, pick-up coils, old video cameras as generators of sound signals (Collins 2006). A similar approach is followed by the circuit benders that collect second-hand toys or cheap sound circuits from garage sales with adventurous attempts of reconfiguring their circuitry. If their experimentation is successful they obtain new functions and expand the sonic possibility of their new acquisition (Ghazala 2005). In video art, Nam June Paik was one of the early artists who repurposed consumer electronics by rewiring televisions in 1963 to display abstract shapes (Parikka 2012). Often referred to by Media Archaeologists, Paul DeMarinis' practice of building digital sculptures is described by Kahn as media deconstruction with the aim of reconstructing new imaginary media narratives (Khan 2010). Wouter van Veldhoven's practice is directed towards audio feedback by building complex networks of old tape machines and mechanical devices (van Veldhoven 2021). Robert Henke repurposed and hybridized Commodore Computers from the ' 80 s with modern circuitry and aesthetics in a contemporary audiovisual performance named $C B M$ 8032AV (Henke 2021). Artists Benjamin Gaulon, Tom Verbruggen and Gijs Gieskes in their Refunct Media expositions, create hybrid digital/analog totems of devices scattered on floors feeding signals into each other (Gaulon 2021). In a similar search for visual materiality the work of Cracking Ray Tube, the duo of James Connoly and Kyle Evans, is directed towards modification of cathode ray tube televisions and computer screens through a hack and crack methodology (Hyde 2020). The duo creates hybrid devices combining analog screens and digital control. Video signals are amplified and sent to speakers for a direct sonic translation of the images displayed on the screen. 


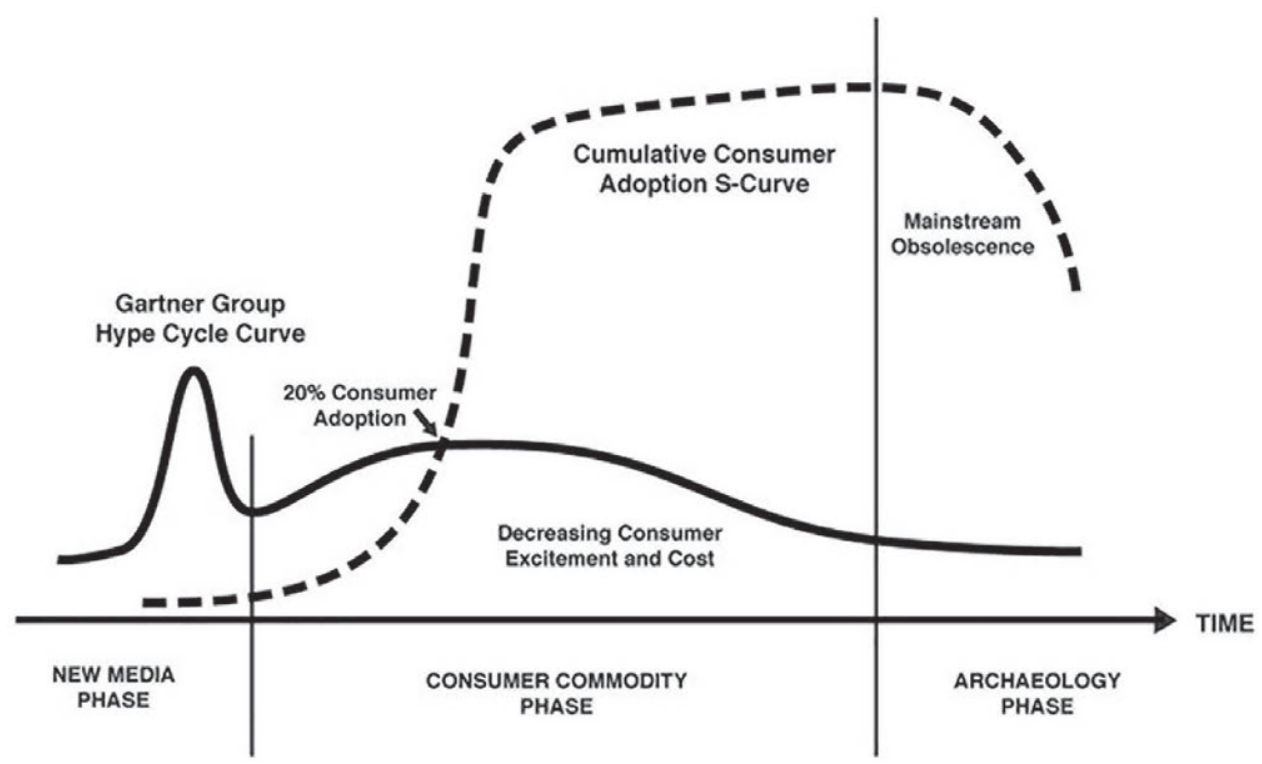

Figure 2. An intuitive graph of the acquisition of media over time. Image courtesy of Garnet Hertz and Jussi Parikka (Hertz et al. 2012).

The tinkering of junk media appeals especially to the artists that cannot afford to stay ahead of the consumer product race by purchasing the ultimate audiovisual machine every six months (Holzer 2019). When the majority of consumers do not find a product appealing anymore it can be easily acquired by artists for dissection and experimentation. Media Archaeology becomes a way for artists not to embrace new trends but to reflect upon them (Herz et al. 2012).

\section{Media archaeology-based visual music}

My artistic practice intersects the methodology of Media Archaeology with the aesthetics of Visual Music in the form of live performances and installations. Since 2014 I have created works for laser projectors and progressively explored other devices that construct images from horizontal and vertical analog signals such as vector monitors (from 2016) and oscilloscopes (from 2018). Some of the works have been released in fixed form, as abstract films screened in international festivals of expanded cinema and new media art.

A common characteristic found in all of my work is the creation of images using audio signals that are sent identically and synchronously to the loudspeakers to generate sound and to displace a light point vertically and horizontally to create images. The movement of the point is so fast that our visual system integrates it into a line in the case of lasers and into bidimensional images in the case of vector monitors (Anderson 1993). Once the basic mathematical principles of correlating horizontal and vertical 
signals to create a trajectory are understood, the task of creating new and interesting results is only limited by the artist's imagination. The premise behind the work is that the audience sees and hears the same electrical signal at the same time creating a synesthetic experience that binds the visual and aural perception of the public to generate new sonic and visual experiences. The direct conversion of sonic vibration into light movement creates a coherent multimodal medium rendering unnecessary any interpretation or arbitrary translation of one domain to another.

\subsection{Laser Drawing}

I started working with lasers in 2014 in preparation of a piece commissioned by Amsterdam Dance Event 20154. In what later became (un)focussed 5 I decided to laserproject the spectrum of the performers' brain activity in the shape of a circle around their bodies.

At that time, lasers were controlled by computers with proprietary software via analog to digital converters and a typical laser show mostly consisted of a play-back of pre-recorded frame-based animations. The International Laser Display Association (ILDA) file format for these animations contains definitions of shapes as a set of points coordinates, every point is associated with one color and every segment connecting two points has only one possible color. The definition of point-based animations, useful for commercial applications aiming at drawing the logo of a brand with sharp edges, often resulted in standardized aesthetics with rigid laser movement and color changes. The live interaction was very limited: some consoles could add shape rotation and color modification (VectorSynthesisForum 2021).

The ILDA standard plug on every laser requires two signals to activate the electrical engines (galvanometers) that deflect the laser beam with two mirrors horizontally and vertically. Three other signals define the beam color as levels of red, blue and green. With a cable hack I was able to input voltages directly from my computer soundcard into the ILDA plug to create shapes at a much higher sample rate $(192 \mathrm{KHz})$ than the proprietary laser software. The possibility to use curves and functions instead of pre-determined fixed point positions, allowed me to reach a completely different laser aesthetic, with much smoother shape transitions, and continuous color modulation unattainable with the rigid ILDA file system. Moreover, I did not need to purchase a laser digital-to-analog converter, I could route the signals to the speakers to listen to the sound connected to the image directly, and I extended the on-the-fly interactivity using customizable real-time audio software.

Considering the aesthetic advantage brought by transitioning from frame-based animations to independent functions, after (un)focussed I decided to explore the potential of a completely analog audiovisual performance deflecting the laser beam with

${ }^{4}$ On ADE15 Magazine https://issuu.com/amsterdamdanceevent/docs/ade_magazine/103.

5 (un)focussed @Warp Amsterdam, https://vimeo.com/jestern/unfocussed. 
the signals from my modular synthesizer instead of the computer. I wanted to diverge from the ubiquitous aesthetics of digital projections and define a personal signature for an audiovisual show. Familiar with Bute's pioneering work on Lissajous figures from the '30s and Laposki's from the '50s (Lissajous 1857), I was convinced that there was much more to explore in terms of laser imagery. My first attempts relied on well known parametric functions to expand my dictionary such as chaos attractors, platonic solids, spherical harmonics, supershape, hypotrochoid, etc. Apart from the visual aspect, I was fascinated to listen to the synthesized sound resulting from each shape. Originating mostly from unfiltered oscillators and geometric relationships between horizontal and vertical axes, the resulting audio is rather harsh. However, expressive compositional results can be obtained through audiovisual modulations following a spectromorphological approach (Smalley 1997). For example, amplitude modulation of the audio waveform equals a modulation in image size. When designing an image, I kept sound in mind in order to create interesting results independently in both senses: visual and aural. All these considerations converged into my performance Laser Drawing (Novello 2019).

During my explorative work, I realized how through the direct visual representation of sound I could more easily perceive and focus on details that otherwise would have been less obvious, such as phase relationships, filtering, waveshaping, etc. To stay coherent with my explorative methodology, in Laser Drawing I maintained the direct sonification of the image signals: routing the horizontal signal to the left audio channel and vertical signal to the right audio channel. In an era of pervasive image over-stimulation where our aural attention and listening skills are often untrained (Hutmacher 2019), by keeping a direct translation of image into sound, I aim at providing the audience with the possibility to perceive smaller sound details. I call it visual listening, extending the concept of deep listening by Pauline Oliveros in the case of Visual Music (Oliveros 2005).

To diversify from the research and style of previous laser artists such as Edwin van der Heide, Robert Henke, and Robin Fox, Laser Drawing is entirely controlled by my modular synthesizer and requires no haze. Haze enhances and gives depth to minimal images, however it can make it difficult for the audience to appreciate the details of complex and dynamic images.

The live performance is approached as an audiovisual improvisation that explores a modular synthesizer patch conceived as an extension of the Syntheshape diagram by Mitchel Waite (Waite 1947). The system required to create such a patch is very similar to the approach I used for years for my live audio improvisations on my modular synthesizer. The central node of the patch is a phase displacement oscillator that allows creating phase shifts between horizontal and vertical axes with different waveforms at low and high frequencies. It is an essential functionality in order to rotate figures and draw, for example, a perfect circle, a square or an equilateral triangle. For the creation of more complex shapes, I combined four oscillators, grouped in two pairs: for horizontal and vertical oscillation and for frequency modulation. Each oscillator can output sine, triangle, sawtooth, square or a combination of these elementary waveforms. All four oscillators can be cross modulated for extreme circular feedback. 

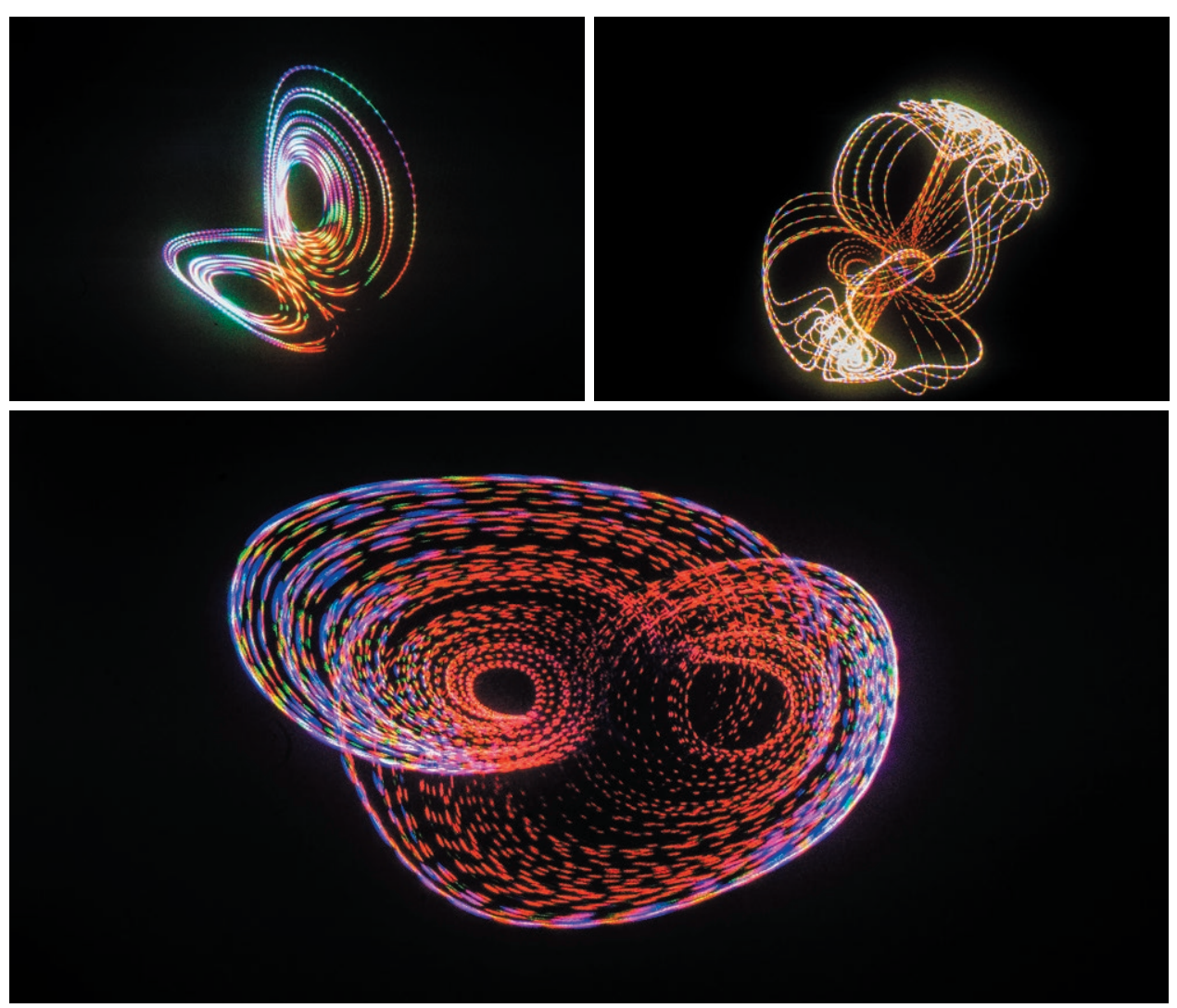

Figure 3. Laser drawing patterns.

A fundamental aspect of a vector graphic system is the possibility to create integer frequency ratios between horizontal and vertical oscillators, both for the shape definitions and for their rotation. Harmonic (integer) ratios generate stable figures with as many lobes as their ratio values (e.g., an octave generates two lobes; a fifth generates three lobes, etc.). Inharmonic (non-integer) ratios create figures that spin with proportional speed to the frequency difference between horizontal and vertical oscillators. In my experience a small deviation from perfect integer ratio is always the most pleasing sensory experience: a slow dance between stability and chaos. The small movement introduces the illusion of a two dimensional image rotating in a tridimensional plane. In the audio domain this effect translates as an interference beat, a mellow aural massage.

In order to quickly shift from stability to chaos passing through a slow dancing image, I designed an intuitive sequencer composed of a low frequency oscillator (LFO), passing through an offset- attenuator, then through a quantizer, and several analog-shift-registers (ASR). The LFO defines the speed of sweep through a range of frequencies defined by the offset-attenuator. The quantizer, typically employed to select among a set of desired pitches, collapses the frequency range into fixed integer frequency ratios. The ASRs distribute the fixed ratios to the four oscillators which 

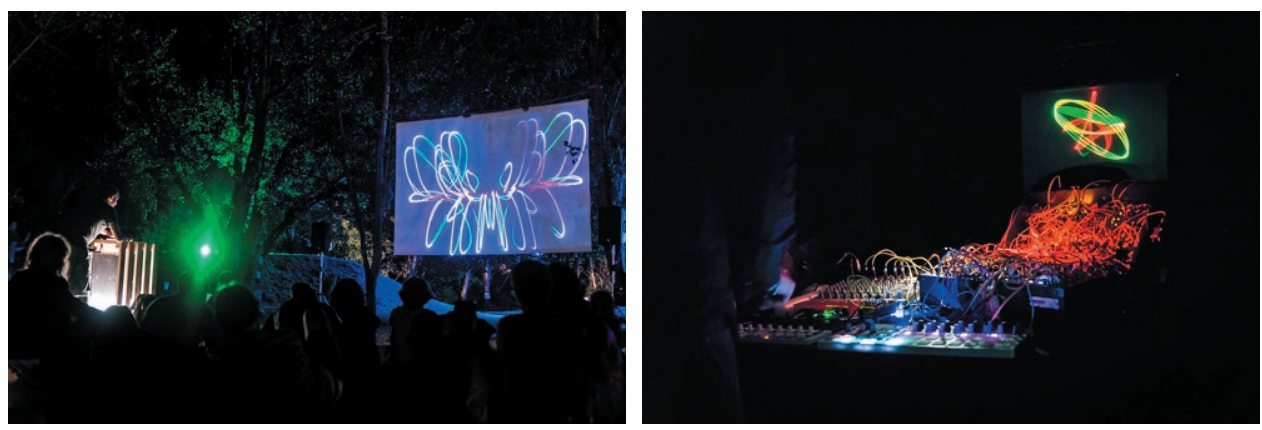

Figure 4. Laser Drawing, typical setting and live setup consisting of a modular synthesizer, a drum sequencer, mixer, a Bela board to generate some digital laser shapes, and a MIDI controller. Photo by Erin McKinney - Courtesy @Erin McKinney.

can be manually detuned by the performer at will. The ASRs are triggered by a selfmodulating clock (composed by a square LFO, a slew-limiter, and a sample-and-hold) to adjust the amount of random temporal-deviation to the regularly time-spaced triggers. With such an architecture I can precisely control the harmonic ratios of my oscillators for stable shapes, slightly detune each oscillator manually to create slow morphologic evolutions, define rhythms in a quantized way, and introduce progressive stochastic changes with a control on the density of events. Each oscillator pair passes through a voltage control amplifier to resize the image, and filters to smooth image corners and add spirals in the case of extreme resonance settings. Both voices have a waveshaper/folder to create more complex forms and a ring modulator for the multiplication of two signals. The four-quadrant multiplication allows drawing a (typically smaller) shape onto a (larger) frame (Waite 1980).

As for the case of acoustic music instruments, improvising with such system requires a muscle memory to rapidly transition through different sonic scenarios: from drones to rhythms, from harmonic ratios to inharmonic experimentations, and quickly find a way to modulate each specific parameter by adding new patch chords. The performance structure typically takes the audience through the exploration of several sonic synthesis paradigms and their visual implications. A usual departure point is simple Lissajous drones to acquaint the audience with the process of visual listening. The performance progressively shifts to more complex signals: rhythmical Lissajous transitions, dephased Karplus-Strong (that creates organic laser bubbles) (Karplus et al. 1983), glitchy Japanese-style sinusoid-noise patterns, to rhythmical techno-influenced explosions using bit reduction and polygonal synthesis (Hohnerlein et al. 2016). A digital sampler contains short samples of pre-recorded material that ease the transition in the cases when many knobs have to be reset for the next section. Reverb and delays are in general avoided to keep the shape clean and visible for the audience.

The position of the performer in a laser show has followed different approaches. In his performance Single Origin, Robin Fox stands on the stage next to his laser and projects the beam towards the audience and on the theater walls. Edwin van der Heide sits inside the projection space away from the lasers, trying to avoid the position on an 
elevated stage. To avoid security issues connected to lasers, I perform in the middle of the audience with a laser on a pedestal far above the audience's heads, projecting on a wall or a white screen positioned in an area where there is no public. I keep the laser switch at arms reach in case I need to switch it off. I chose this position to monitor what I am projecting and to allow the audience to observe where the light beam comes from, realize that I am actively modifying it with the machinery next to me, and follow my actions and their immediate visual result.

As with every laser show, documentation of Laser Drawing is difficult, it has to be experienced live and filmed versions have always proven to be inadequate. This is primarily due to the high dynamic range of laser light, impossible to capture by CCD sensors. Moreover, being generated by a moving dot, to the human eye the laser image is an infinite line; digital camera frame rate cuts the line into many segments, disrupting the image continuity and beauty.

Laser projectors are more efficient than digital ones because laser light concentrates all energy in one point. With less power consumption than a digital projector, a lowend laser projector creates very bright images projecting as far as hundreds of meters, is less expensive, and more compact making it ideal for touring.

Thanks to these characteristics, over the last five years I have performed my show more than fifty times around the world, from The United States of America to Asia, in international festivals (Serralves in Festa (Porto), Inner Spaces (Milan), Highlight Festival Delft), in official Art Institutes (Glasgow Contemporary Art Museum, Maryland Institute of the Arts, Conservatory of Turin, Sussex University, Rome University of Fine Arts), as well as in experimental secluded locations such as the Mekong Jungle in Vietnam. I have also presented my work in specialized festivals of visual music such as the biennial Vector Hack organized by Ivan Marušić, Derek Holzer and Chris King, Seeing Sound organized by Joseph Hyde at the University of Bath UK, Intermediale in Poland, Media Festival South Bend in Indiana, USA to name a few. Possibly inspired by the discourse of Media Archaeology, I particularly enjoy the combination of new and old in regards to the location: I projected lasers on old village walls in the Puglia region of Italy, on traditional housing in Vietnam, in dark factory warehouses in the Netherlands. I also enjoy projecting from unusual locations, for example during the COVID-19 pandemic I created several impromptu street performances from the rooftops of buildings in the city of Trieste, Italy ${ }^{6}$.

To create easily accessible interfaces for younger generations and non-technical enthusiasts, I have developed different tools combining tablets ${ }^{7}$, micro-controllers such as Bela and Arduino and micro-computers such as Raspberry Pi to drive lasers (Bela 2021, Arduino 2021, Raspberry Pi 2021). I have been invited to give workshops to various groups: from children to adults, from visual artists to hackers, and composers in several countries (Maryland Institute of the Arts, Conservatory of Turin, Sussex

${ }^{6}$ Impromptu Rooftop Laser Performance during COVID-19: https://vimeo.com/jestern/rooflaser1, https://vimeo.com/jestern/rooflaser2, https:/vimeo.com/jestern/rooflaser3, and https://vimeo.com/ jestern/rooflaser4.

7 The installation for young laserists, Lasertablet: https://vimeo.com/jestern/lasertablet. 


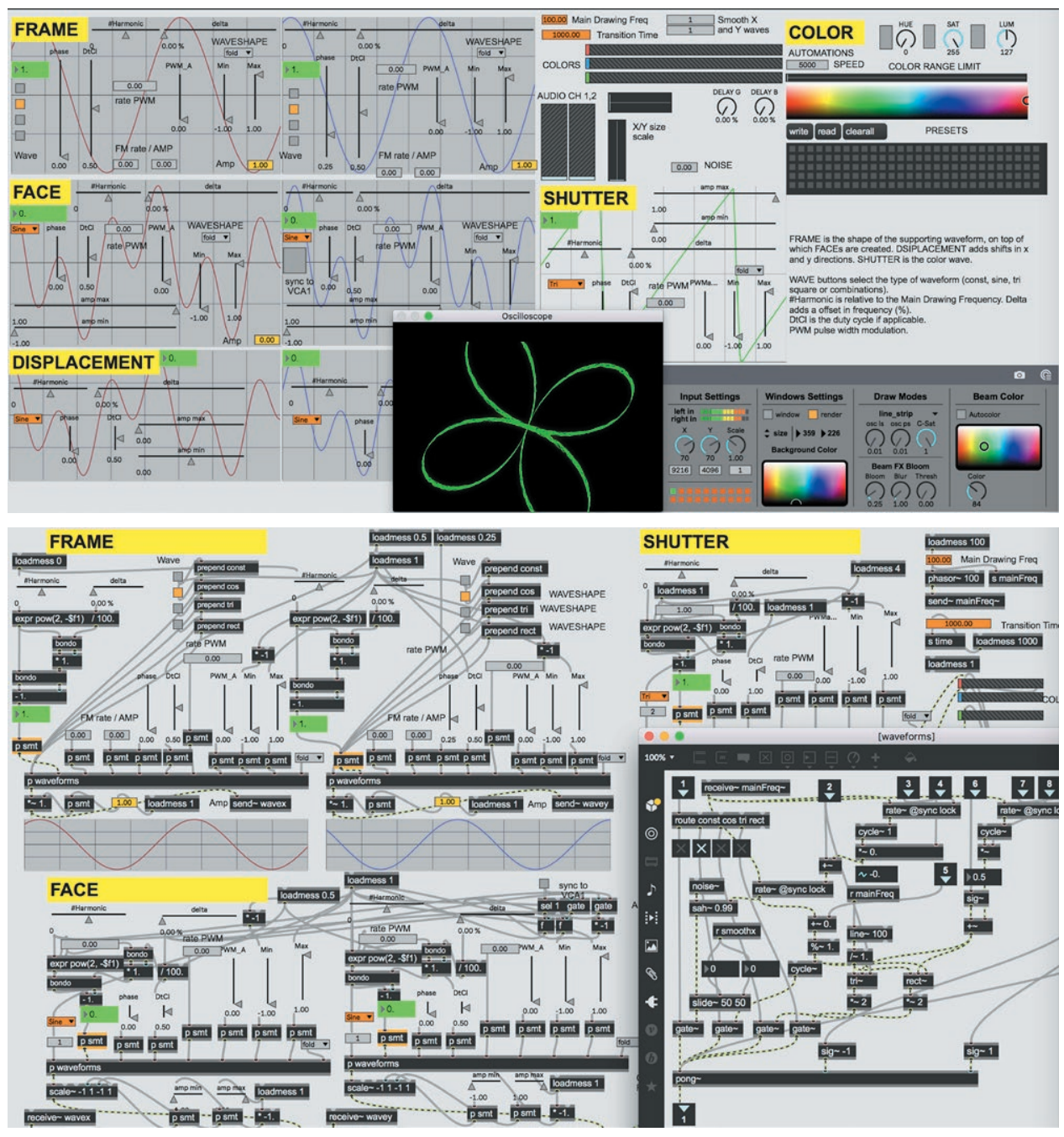

Figure 5. Example of Laser Drawing patches for Max/MSP released as free software on the author's github.

University, Rome University of Fine Arts, Trieste Maker Faire). At the intersection of multiple disciplines, these workshops provide, through hands-on experience, a theoretical and practical introduction to electronics, optics, coding, laser physics, and the more philosophical topics connected to the social impact of technology, new media and obsolescence. The laser patches developed in Max/MSP during my first few years of research to prototype most of my initial laser works are available for free on github, with the intention to inspire new laser enthusiasts to develop their own style ${ }^{8}$ (DoubleOscillator.maxpat). Together with artist and hacker Luka Frelih, I organized

${ }^{8}$ Laser Drawing Max/MSP patches released on github: https://github.com/jestern77/LaserDrawing. 
a workshop on how to build an inexpensive and limited laser projector with less than ten euros using the circuit of an old hard drive (Frelih et al. 2020). The musician and designer Aimo Scampa, and I are developing an inexpensive Raspberry Pi-based digital laser synthesizer that will be released commercially in the near future.

\subsection{Black Light}

In the last year, inspired by and dedicated to the work of Aldo Tambelini, I created a performance for laser light on a photosensitive canvas: Black Light ${ }^{9}$. The UV part of the laser spectrum leaves a trace on the $8 \times 2 \mathrm{~m}$ surface that lasts for ten seconds to one minute, depending on the chemical composition of the photosensitive compound used. This effect allows for a different style of light painting compared to Laser Drawing. The beam movements can be slow and the persistence of the trace allows the superposition of shapes for the creation of complex disappearing worlds.

\subsection{Vectrex Works}

In search of more complexity I experimented with vector monitors that can display the same mathematical equations as lasers but reach a higher beam speed thus intricate imagery. The deflection of the light beam in lasers happens through the use of mirrors moved by small engines. Fast deflection speed at high angles causes overheating and failure. Sharp corners also strain the engines. The sound and visual material available for the artists is mostly composed of curves, which can also be rather complex such as the case of spherical harmonics. However, to reach more complex results, faster deflection is needed. Deflecting its beam through an electrical field instead of a physical object, a CRT monitor can handle higher frequencies and sharp corners. Following an historic thread by Lars Larsen, I hacked a Vectrex, a monochromatic game console monitor from the early ' 80 s. Decoupling its game engine from the monitor, it is possible to input three signals and control the horizontal and vertical position, as well as the intensity of the beam (Larsen 2012).

In order to document my exploration of the medium, in the last five years I have produced several performances and fixed abstract audiovisual pieces. I started with only analog technology to drive the Vectrex: Celestial Harmonies ${ }^{10}$, a video composition of 13 minutes aiming at extending the classic curvy Lissajous figures with more noisy/glitchy and stroboscopic patterns. In Celestial Harmonies the reference to Optical-Art (or better its extension into a kinetic light Op-Art) is visible through the exploration of Moiré patterns across the three dimensions: horizontal, vertical and intensity. For the subsequent works I took inspiration and formulas from the litera-

9 Black Light, UV laser performance: https://vimeo.com/jestern/blacklight.

${ }^{10}$ Celestial Harmonies, https://www.vimeo.com/jestern/celestialharmonies. 
ture on computer graphics: 3D solids and rotations, multiplexing, flyover-blanking, the use of perspective, movable or parameterizable light sources and shadows, adding gravity and Newton's laws of motion ${ }^{11}$. For these more precise works I had to shift from my modular synthesizer to digitally generated signals via Max/MSP through a sound-card to maintain exact phase relationships and frequency tuning ${ }^{12}$. As in the case of Laser Drawing, for all Vectrex studies the resulting sound has as much as an important role as the image.

CRT screens do not have digital outputs for video capture so a proper documentation of these works requires effort and understanding of the medium. The artist needs to record the screen with a camera, a process called rescanning. This practice is an essential part of live performances: re-projecting the small screen for the audience. Learning the proper capturing technique, in particular adapting the camera sample rate and shutter angle to the beam speed, is essential to the process.

My research with the Vectrex monitor produced two installations. The first is a half digital/half analog contemplative hybrid object combining the fluidity and luminance of analog beams and the control of digital circuitry ${ }^{13}$. A Bela computing platform generates the audio signals to create the shapes on the monitor. The same signal is amplified and played through the built-in speakers. With this work I reflect on the temporality of technology: the meeting of old and new and how the old is reinterpreted with new aesthetics. The installation is contemplative, in reaction to the recent trend of interactive installations requiring the audience to repeat simple actions and reducing them to mechanisms instead of independent and thinking individuals. Through contemplation I aim at re-tuning the audience to the present moment, allowing them to observe the beauty of a phenomenon that evolves by itself.

The second installation is a study on stereoscopic perception. The work uses two Vectrex screens to display the same 3D scene from two different perspectives. The images are generated to simulate the different points of view from the observer's right and left eye. It is aimed at reinterpreting an old methodology, the Wheatstone design, from the end of the nineteenth century with modern but also obsolete tools (Funk 2012).

\subsection{Scan Processing}

One of the intrinsic limitations of Vectrex monitors is the low bandwidth of the input signals they can handle. The audio coils, used in the construction of the screen to deflect the beam, did not need high resolution for gaming purposes. Above a few $\mathrm{KHz}$ the lines become distorted, overheating the screen circuitry. For the creation of more complex imagery, monitors with higher resolution are needed: higher number of scan

11 The author's Vimeo channel, https://www.vimeo.com/jestern.

12 It is desirable in this case to have a DC-coupled soundcard, e.g. a sound-card that can output constant signals to create more complex imagery. A non DC-coupled soundcard, or AC-coupled, can in general be simply hacked to become DC coupled.

13 Celestial Harmonies Installation, https://vimeo.com/jestern2/celestialharmonies. 

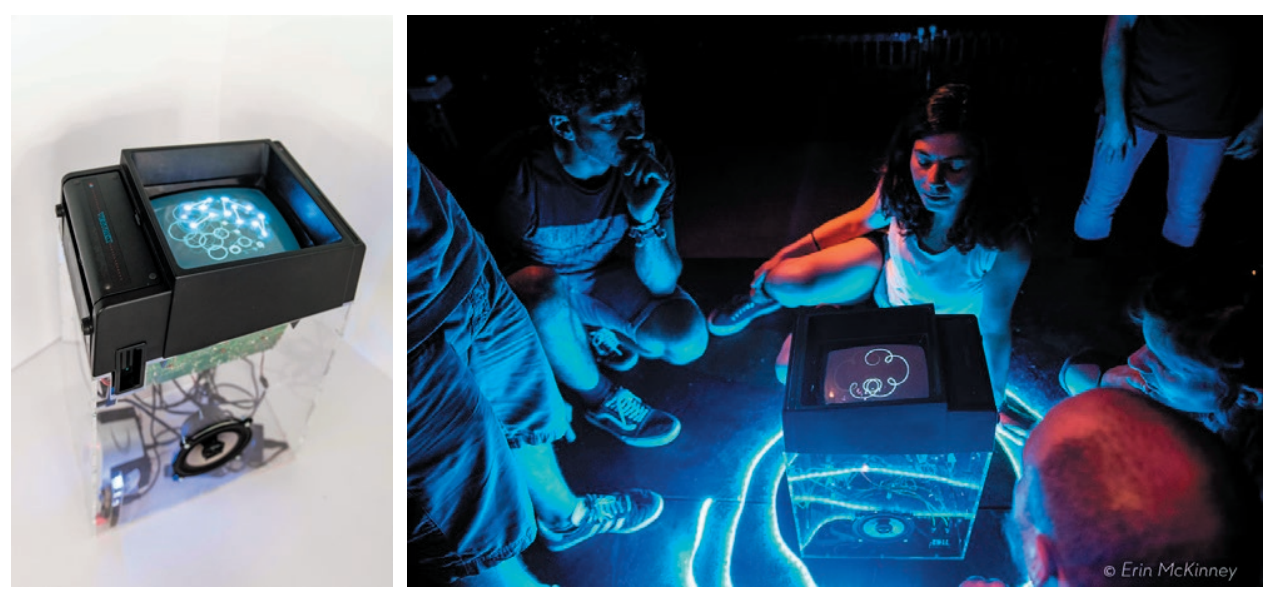

Figure 6. Celestial Harmonies - hybrid contemplative installation using a vector monitor from a modified Vectrex game console from the ' 80 s, a Bela computing platform, speakers, and amplifying circuitry.

lines require faster deflection driver coils. Vector screens with such characteristics are for example the Tektronix six hundred series or the Leader LBO51, with a bandwidth of $3 \mathrm{MHz}$ for the drivers. These screens have removable graticule for those who prefer a cleaner aesthetic during capture.

Woody and Steina Vasulka are the artists who pushed the boundary of deforming the bidimensional TV image frame (raster) into 3D "time/energy" objects through what they call scan processing or raster processing (Vasulka et al. 1992). Instead of the typical video signal that can be displayed on an old fashion television, scan processing requires three signals (horizontal sweep, vertical sweep and beam intensity) to shape the frame of the image itself, and a vector CRT monitor, or oscilloscope to be displayed. The look of the scan processing effect developed by the Vasulkas has been so influential that several digital software have tried to emulate it (Marini 2009, Crx 2009).

To continue the experiments and theoretical framework laid by the Vasulkas, I have created several works using scan processing techniques. My objective was to generate and deform a single object and extend Vasulka's approach with the multiplexing technique having two or more independent raster objects ${ }^{14}$. I focused in particular on superposition of noise patterns and video feedback fractals on specific raster shapes such as plane, sphere, cube and cylinder ${ }^{15}$. In an attempt to create a 3D method for sound visualization, I used scan processing to display a song spectrogram dynamically extruding from a plane ${ }^{16}$.

Even at the highest possible sample rate of $192 \mathrm{KHz}$, the signals generated by a computer and a soundcard cannot reach the necessary scanline resolution of a system

\footnotetext{
14 Juggling Planes, short abstract movie https://vimeo.com/jestern/jugglingplanes.

15 Feedback Plane \#2, short abstract movie https://vimeo.com/jestern/fbplane2.

16 Last Minute Pre-Dawn Chaos - Part III, short abstract movie https://vimeo.com/jestern/lmpcIII.
} 
such as PAL which has 25 frames per second with 512 lines. For my last works I assembled an analog modular video synthesizer system composed of $L Z X$ Industries Cadet modules inspired by functionalities of the historic Rutt-Etra synthesizer built by Steve Rutt and Bill Etra (Vasulka et al. 1992, LZX Industries 2021). In the first studies I added video feedback to control extrusion on planes in a Rutt-Etra-esque aesthetic with image properties controlling sound synthesis: the level of image brightness opened gates in a feedback modular synthesizer patch and a measure of frame difference affected several parameters in a granular synthesis Max/MSP patch ${ }^{17}$. In these early studies, despite the synchronous connection between video and audio, there is no direct translation of signals between video and audio as in the strict visual music definition suggested for this article. In the case of scan processing it is difficult to choose which part of the signals to sonify through the speakers. The high frequency imposed by the construction of the image $(50 \mathrm{~Hz}$ ramp signals vertically and $15 \mathrm{KHz}$ horizontally) is a strong limitation to the expressive possibility of the artist. I followed three alternative strategies: selecting instead a control signal to be sonified through the loudspeakers, a signal of the overall intensity or modulation of the shapes to control a filter of the underlying image drone, and use reverb to mellow the harsh output.

\subsection{Combining Laser light and CRT images: Inspirals}

To continue the work of Woody Vasulka and his research towards time/energy objects, in the last year I developed a novel technique that fuses the shapes obtained from two coherent but different light sources into a complex 3D object: the laser projections and the images from the CRT screen. The presence of two light sources adds an extra layer of depth to the image. In my last live AV performance Inspirals the monochromatic CRT monitor is used to form the complex and detailed shapes while the more intense and colorful laser beam is used to add a second layer that seems to radiate out from the image. Merging together the CRT and Laser image requires perfect synchronization: a Pure Data patch running on a Bela platform and controlled via a MIDI interface creates precise clocking and phase displacements of the two beams. The performance draws inspiration from and vaguely depicts deep space fauna: binary stars, lenticular galaxies, quasars, black holes, and curved spacetime continuum ${ }^{18}$.

\section{Conclusions}

This article describes the connection of Media Archaeology and Visual Music in my artistic practice that repurposes obsolete devices to investigate the connection between light and sound. I revive and modify tools from our analogue past: oscilloscopes, early

17 LZX-based feedback scan processing: digital video feedback https://vimeo.com/jestern/cadetfeedback, and analog https://vimeo.com/jestern/feedackscanprocessing2.

18 Inspirals, https://vimeo.com/jestern/inspirals. 

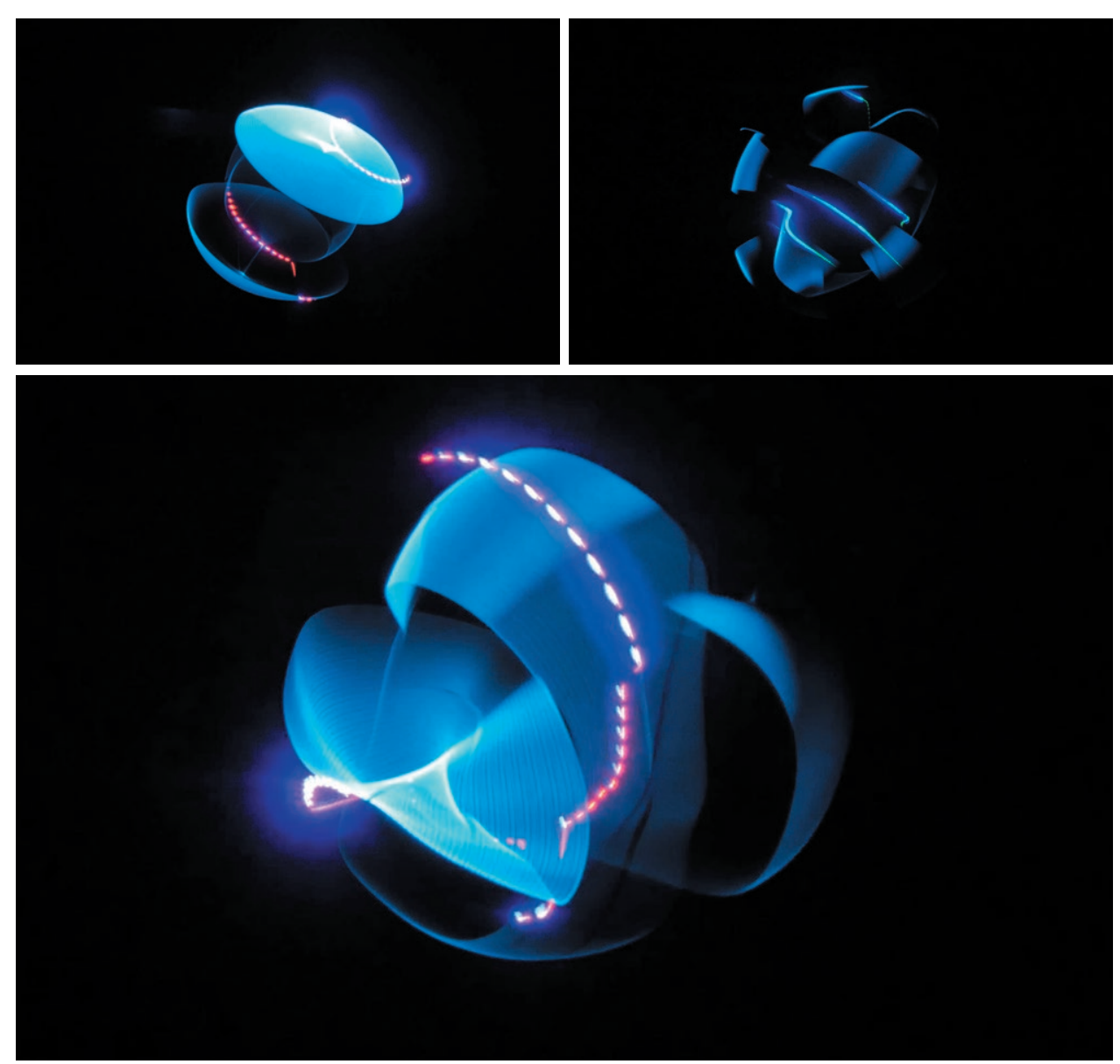

Figure 7. Stills of Inspirals, an AV performance using a novel technique to superpose laser light to CRT images.

game consoles, analogue video mixers, and lasers. I am attracted to their intrinsic limitations and strong 'personalities': fluid beam movement, vibrant light, infinite resolution, absence of frame rate, and line aesthetics. A central part of my art is the beam of light of these devices, made of photons in the case of lasers and electrons in the case of CRT monitors, that I deflect to compose images. Because of the difficulty of documenting the vibrance of these light beams and in order to expose the audience to the full aesthetic potential of my practice, I am mostly interested in releasing my work in the form of contemplative installations or live performances.

The premise behind all my work is the synthesis of image and sound from the same identical signal. The direct and simultaneous translation of a signal into sound and image creates a synesthetic experience for the audience connecting synchronously the aural and visual systems. This aesthetic choice allows the audience to explore sound with the help of its visualization. Predisposed towards visual stimulation, contemporary society is less and less attentive to the aural sphere (Hutmacher 2019). The direct 
translation of the same signal into light and sound envelopes the audience in synchronous information that reveals underlying geometric properties of sound: frequency ratios, de-tuning and phase shifts, which would go mostly unnoticed, are amplified through their visual counterpart. The audience listens in a new way to the sound details experiencing what I call visual listening.

The simultaneous control of two mediums frees the performer from the arbitrary choice of what audio material to attach to the visuals. The artist needs to develop a personal dictionary of signals connecting the two senses. They can then shift weight during the performance, passing from moments in which audio is more relevant to moments where video takes precedence, allowing for a dynamic change that keeps the audience's attention alive. Experience is necessary to predict the impact that a change of signal has on each medium, image and sound, and how to transition between parts during the performance. The limitations imposed by the analog technology, for example the fact of creating all images using only three signals, becomes a liberation compared to the disorientation that the infinite possibilities of the computer create.

The paradigm shift that society embarked on in the ' 80 s towards personal computers and digital technologies produced a loss of media materiality and the impossibility to interact, operate, and repair machines: a consequence of what some identify as planned obsolescence. Due to this phenomenon society has created an enormous amount of discarded devices, among which the CRT monitor is one of the main symbols of the growing toxic waste. What can we do with old tools, in an era in which human overpopulation forces us to rethink our technology, where the accelerating effects of climate change run parallel to the unstoppable race for technological development, which is often considered a solution, rather than a cause, of the inevitable environmental catastrophe?

Digital tools promised greater aesthetic possibilities while creating a «culture of restrictive interfaces and preset creativity» often driven by passive and uncritical consumption (Connolly et al. 2014). However, in the avant-garde, digital audiovisual performances reached a saturation and many artists are diverging from the mainstream in search for a personal approach through materiality or retro aesthetics (Zaarei 2020, Parikka 2012).

The decision of working with old devices opens up a relatively less crowded and free field of aesthetic exploration compared to digital performances. The absence of mainstream frees the artist from the concept of 'making mistakes' as there is no established aesthetic. Besides their different aesthetics, there is a charm in repurposing obsolete hardware for the socio-political gesture of counterbalancing the race of acquiring the latest/fastest technology. From being unused and thrown away, obsolete machines acquire a new potential thanks to the possibility of being opened and their circuitry being reconfigured. The possibility to expose their inner workings allows the artist to use the materiality of these old devices to challenge technological, aesthetic, social and economic assumptions of the present. Instead of becoming deadly chemical waste, the device is reborn at the center of the artistic production. It is culturally viable and a symbol of obsolescence at the same time. 
In this paper I describe the practice and the aesthetic potentials of few analog and digital hybridized systems to generate new sonic and visual experiences. In particular the combination of digital control and fluidity of analog light beams often creates strangely familiar but aesthetically new results. Together with other philosophers such as Zielinski (Zielinski 2006), other artists such as DeMarinis (Parikka 2012), Connolly/Evans (Connolly et al. 2014), I believe in the power of combining both worlds into new hybrid methodology and objects. After many years spent coding I felt an intrinsic need for a return to materiality, to open and dissect, solder, interpret and rebuild my way.

Through hacking and circuit bending the contemporary artist can resurrect disregarded machines and take advantage of their retro-aesthetic for new performative results. In particular I describe the case of Celestial Harmony, an installation of media archaeology based visual music, composed of a hacked CRT screen of a '80s game console driven by the outputs of a digital micro-controller to create generative light structures. It is a contemplative installation to re-tuning the audience to the present moment and allow them to enjoy the beauty of a phenomenon that evolves by itself. Media Archaeology as artistic practice exposes the audience to zombie devices allowing them to reevaluate their possibilities and realize the potential contained in old technology. It is a retrospective on technologisation: what old means, and what value the new really adds, in order to stimulate and reconsider the potential of obsolete devices and creatively imagine the potential contained within discarded analog technology when repurposed through digital hybridization.

The practice of repurposing obsolete technology and aesthetics requires a new methodology for the artist who becomes more of a live explorer in the sense of an improvised-music ensemble than a performer of a detailed and written score with preset algorithms and pre-rendered parts. I believe in the performative added value of exposing myself to the risk of failure of material instrumentality. The shapes I create through light, often need re-tuning and on-the-fly adjustments, making the performance more of a light-juggling show than a predetermined and fixed act. I keep some safe zones and parts that I choose to repeat which are aesthetically captivating, however almost half of every liveset is improvised for my own inspiration and to differentiate each performance. Through improvisation the audience can better understand the rules at play and recognize the limitations and risks taken by the artist. Another essential skill that the artist has to develop in this practice is the ability of excavating the internet in search for old devices: without the proper archaeological work it is impossible to achieve a new/old aesthetic. In the case of rare items it is not uncommon for the artists to purchase a broken device, relying on their technical knowledge, or the one of the community, for reparation. The artists are in this sense not only composers or coders, but need to also know basic optics, electronics, engineering, master soldering, and circuit designing.

New aesthetics also require a new attitude from the audience to adapt to a new medium. In general visual music is an experiential perception requiring no explanation: the presence of one signal driving both sensory stimulations becomes clear immediately through the synchronous and synesthetic connection between 
vision and aural perception. The listener is then more prone to accept the unusual sounds required to create the complex shapes understanding the inseparable nature of sound and image.

\section{Acknowledgements}

This article is dedicated to the memory of laser artist Ronald A. Pellegrino (19402021).

\section{References}

Abbado, A. 2018, Visual Music Masters: Abstract Explorations of Past and Present Artists, Skira. Anderson, J. and Anderson, B. 1993, The Myth of Persistence of Vision Revisited, "Journal of Film and Video", 45(1): 3-12.

Arduino 2021, official website, <URL: https://www.arduino.cc/> (03/21).

Bela Board 2021, <URL: https://bela.io/> (03/21).

Bernard, J. W. 1986, Messiaen's Synaesthesia: The Correspondence between Color and Sound Structure in His Music, "Music Perception", 4: 41-68.

Bernstein, D. 2015, John Cage’s Cartridge Music (1960): 'A Galaxy Reconfigured', «Contemporary Music Review», 33(5-6): 556-569.

Cadoz, C. 1988, Instrumental Gesture and Musical Composition, ICMC 1988 - International Computer Music Conference, Germany, pp.1-12, <URL: https://hal.archives-ouvertes.fr/ hal-00491738/document>.

Carsten, N. 2021, personal website, <URL: http://www.carstennicolai.de/> (03/21).

Collins, N. 2006, Handmade Electronic Music, Routledge.

Connolly, J. and Evans, K. 2014, Cracking Ray Tubes: Reanimating Analog Video in a Digital Context, «Leonardo Music Journal», 24: 53-56.

Crx, 2009, Rutt Etra Jitter Video Synth For Free Download, <URL: https://reaktorplayer.wordpress. com/2010/08/17/rutt-etra-jitter-synth-for-free-download/> (03/21).

Cytowic, R. E. and Eagleman, D. M. 2009, Wednesday is Indigo Blue: Discovering the Brain of Synesthesia, pp. 100-112, MIT Press.

Dann, K. T. 1998, Bright colors falsely seen: synaesthesia and the search for transcendental knowledge, Yale University Press.

deWitt, T. 1987, Visual Music: Searching for an Aesthetic, «Leonardo Music Journal», 20(2): 15-122, MIT Press.

Evans, B. 2005, Foundation of Visual music, "Computer Music Journal», 29(4): 11-24, The MIT Press.

Evers, F. 2020, The Academy of the Senses, Synesthetics in Science, Art and Education, Art Science Interfaculty Press.

Foucault, M. 1972, Archaeology of Knowledge, Tavistock Publication Ltd.

Fox, R. 2021, personal website, <URL: https://robinfox.com.au/> (03/21). 
Frelih, L., and Novello, A. 2020, Laser Tags Sound Drawings, Making and controlling a laser projector using a CD lens mechanism, Ljudmila website, <URL: https://wiki.ljudmila.org/ Laser_Tags_Sound_Drawings> (03/21).

Friedlander, P. 2021, What is Visual Music?, <URL: http://www.paulfriedlander.com/text/ visualmusic.html> (03/21).

Funk, W. 2012, History of autostereoscopic cinema, "Stereoscopic Displays and Applications XXIII, Proceedings of SPIE-IS\&T Electronic Imaging», 8288.

Garro, D. 2012, From Sonic Art to Visual Music: Divergences, convergences, intersections, «Organised Sound", 17(2): 103-113.

Gaulon, B. 2021, personal website, <URL: http://recyclism.com/refunctmedia2.html> (03/21).

Ghazala, R. 2005, Circuit-Bending: Build Your Own Alien Instruments, Wiley.

Henke, R. 2021, personal website, <URL: https://roberthenke.com/> (03/21).

Hertz, G. and Parikka, J. 2012, Zombie Media: Circuit Bending Media Archaeology into an Art Method, "Leonardo Music Journal», 45(5): 424-430, The MIT Press.

Higgins, D. 2001, Something Else Newsletter No. 1, Reprinted in «Leonardo Music Journal», 34(1): 49-50, MIT Press.

Hohnerlein, C., Rest, M., and Smith, J. O. 2016, Continuous Order Polygonal Waveform Synthesis, «Proceedings of the International Computer Music Conference», The Netherlands.

Holzer, D. 2019, Vector Synthesis: A Media Archeological Investigation into Sound-Modulated Light, Holzer.

Hutmacher, F. 2019, Why Is There So Much More Research on Vision Than on Any Other Sensory Modality?, "Conceptual Analysis, Frontiers in Psychology», 10, Art. 2246.

Hyde, J. 2020, Sound and image : aesthetics and practices, edited by Andrew Knight-Hill, pp. 188-205, New York, Routledge.

Ikeda, R. 2021, personal website, <URL: https://www.ryojiikeda.com/> (03/21).

Jack, O. 2021, personal website, <URL: https://ojack.xyz/> (03/21).

Karplus, K. and Strong, A. 1983, Digital Synthesis of Plucked String and Drum Timbres, «Computer Music Journal», 7(2): 43-55, MIT Press.

Khan, D. 2010, Some artworks by Paul DeMarinis, in Buried in Noise: Paul DeMarinis, Ingrid Beirer, Carsten Seiffarth, Sabine Himmelsbach (eds.), pp. 47-57, Kerhrer Verlag.

Kripper, K. 2021, personal page, <URL: http://signalculture.org/kevin-kripper.html> (03/21).

Kurokawa, R. 2021, personal website, <URL: http://www.ryoichikurokawa.com/> (03/21).

Larsen, L. 2012, Muffwiggler forum, <URL: https://www.muffwiggler.com/forum/viewtopic. php?t=69637> (03/21).

Lissajous, J. A. 1857, Mémoire Sur l'Etude Optique Des Mouvements Vibratoires, "Annales de Chimie et de Physique», 3(51): 146-233.

LZX Industries 2021, <URL: https://lzxindustries.net/> (03/21).

Marini, A. 2009, v002 Rutt Etra 2.0.1, <URL: http://v002.info/plugins/v002-rutt-etra/> (03/21).

McDonnell, M. 2007, Visual music - a composition of the 'things themselves', <URL: https://www. academia.edu/525221/Visual_Music_A_Composition_Of_The_Things_Themselves $>(03 / 21)$.

Novello. A. 2019, LASER DRAWING a synesthetic experience in which you can listen to what you see, and see what you listen to, Proceedings of the Seventh Conference on Computation, Communication, Aesthetics \& X, Italy. 
Oliveros, P. 2005, Deep Listening: A Composer's Sound Practice, Deep Listening Publications.

Ox, J. and Keefer, C. 2008, On Curating Recent Digital Abstract Visual Music, <URL: http:// www.centerforvisualmusic.org/Ox_Keefer_VM.htm> (03/21).

Parikka, J. 2011, With each project I find myself reimagining what cinema might be: An Interview with Zoe Beloff, "Electronic Book Review», 24: 100-112.

Parikka, J. 2012, What Is Media Archaeology?, Polity Press.

Pellegrino, R. 1983, The Electronic Arts of Sound and Light, Van Nostrand Reinhold Company.

Pickering, A. 2011, The Cybernetic Brain, Sketches of Another Future, pp.309-351, University of Chicago Press.

Raspberry Pi 2021, <URL: https://www.raspberrypi.org/> (03/21).

Rogowska, A. 2011, Categorization of Synaesthesia, "Review of General Psychology», 15(3): 213-227.

Shechet Epstein, S. 2018, Creatures of Light: LASERIUM, <URL: http://www.scienceandfilm. org/articles/3115/creatures-of-light-laserium> (03/21).

Smalley, D. 1997, Spectromorphology: explaining sound-shapes, «Organised Sound», 2(2): 107-126.

Smirnov, A. 2013, Sound in Z, Experiments in Sound and Electronic Music in Early 20th Century Russia, Sound and Music/Koenig Books.

Sterling, B., and Jennings, T. 2021, The Dead Media Project, <URL: http://www.deadmedia. org/> $(03 / 21)$.

Tudor, D. 2021, <URL: https://davidtudor.org/Works/rainforest.html> (03/21).

Van der Heide, E. 2021, personal website, <URL: https://www.evdh.net/> (03/21).

Van Helden, A. and Hankins, T. L. 1994, Instruments, "Osiris», 9: 141-156, The University of Chicago Press.

Van Veldhoven, W. 2021, personal website, <URL: https://www.woutervanveldhoven.nl/> (03/21).

Vasulka, W., Vasulka, S., Weibel, P. and Dunn, D. 1992, Eigenwelt Der Apparatewelt: Pioneers of Electronic Art, Ars Electronica/The Vasulkas Inc.

Vector Synthesis Facebook Forum 2021, <URL: https://www.facebook.com/ groups/vectorsynthesis/? multi_permalinks=1596382757237959\&comment_ id=1597367320472836\&notif_id=1616102804398203\&notif_t=feedback_reaction_ generic\&ref=notif> (03/21).

Waite, M. 1947, Projects in Sight, Sound and Sensation, Howard W. Sams \& Co.

Waite, M. 1980, The Oscilloscope Graphic Artist, «Popular Electronics: Electronic Experimenter’s Handbook», pp. 21-25.

Youngblood, G. 1970, Expanded Cinema, Dutton.

Zareei, M. H. 2020, Audiovisual Materialism, «Organised Sound», 25(3): 362-371, Cambridge University Press.

Zielinski, S. 2006, Deep Time of the Media, Toward an Archaeology of Hearing and Seeing by Technical Means, MIT Press. 